\section{Two Cases of Small Non-Polypoid Neoplasia in an Elongated Colonic Polyp}

K. Hizawa ${ }^{1}$, T. Nakahara ${ }^{2}$,

S. Inuzuka ${ }^{2}$, K. Akagi ${ }^{2}$,

R. Shimono ${ }^{2}$, M. Kitamura ${ }^{3}$,

K. Iwai ${ }^{3}$, T. Matsumoto ${ }^{4}$

${ }^{1}$ Department of Internal Medicine, Kyushu Central Hospital, Fukuoka, Japan

${ }^{2}$ Department of Surgery, Kyushu

Central Hospital, Fukuoka, Japan

${ }^{3}$ Department of Anatomic Pathology, Graduate School of Medical Sciences, Kyushu University

${ }^{4}$ Department of Medicine and Clinical Science, Graduate School of Medical Sciences, Kyushu University

\section{Corresponding Author}

K. Hizawa, M.D.

Dept. of Medicine and Clinical Science Graduate School of Medical Sciences Kyushu University

Maidashi 3-1-1

Higashi-ku

Fukuoka 812-8582

Japan

Fax: $\quad+81-92-6425273$

E-mail: hizawa@intmed2.med. kyushu-u.ac.jp

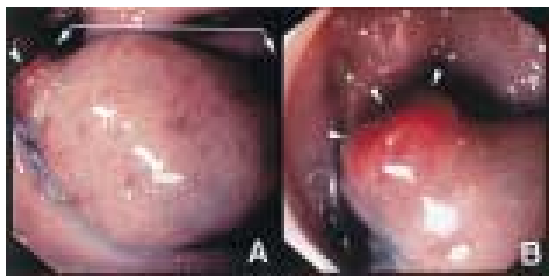

Figure 1 Patient 1: colonoscopy shows a flabby potbelly-shaped pedunculated polyp $(\mathbf{A})$ with a reddish and uneven head (B). The elongated polyp is composed of edematous submucosa and normal colonic mucosa (arrows), containing a depressed form of tubular adenoma (arrowheads)

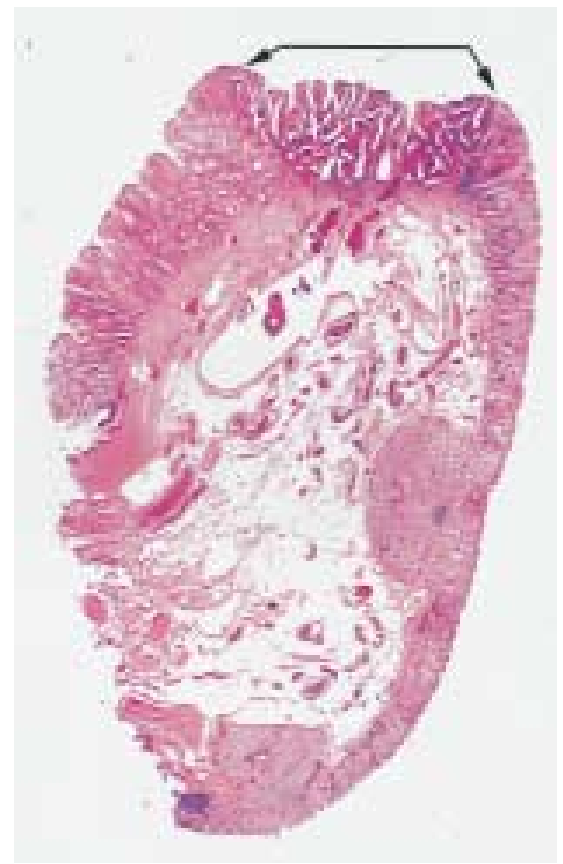

Figure 2 Patient 1: the arrows indicate the tubular adenoma

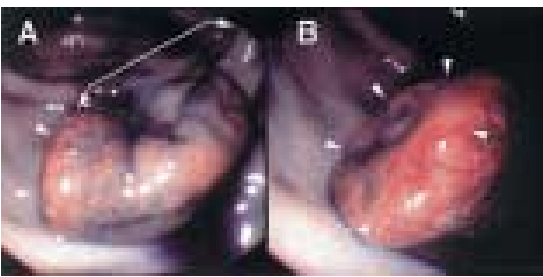

Figure 3 Patient 2: colonoscopy shows an elongated polyp covered by wrinkled normal mucosa (A) with a reddish and slightly depressed surface (B). The polyp has wide areas of hyperplastic epithelium (arrows), and a well-differentiated adenocarcinoma with nonpolypoid growth (arrowheads) invades the edematous submucosa

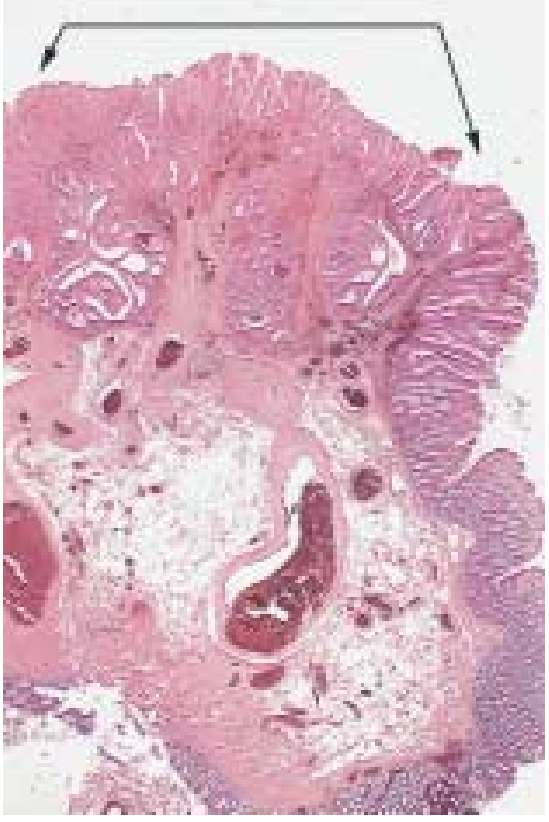

Figure 4 Patient 2: the arrows indicate the well-differentiated adenocarcinoma 